\title{
A Sri Lankan infant with vaccine associated acute flaccid paralysis
}

\author{
P Perera $^{1}$, S Mettananda ${ }^{1}$ \\ Ceylon Medical Journal 2012; 57: 42-43
}

\section{Introduction}

Global incidence of poliomyelitis has reduced by more than 99\% since the Global Polio Eradication Initiative was launched in 1988 [1]. In par with the global trend, Sri Lanka has achieved great success with no reported cases since 1993, but still is not declared as a country which has eradicated polio due to prevalence of polioyomyelitis in neighbouring countries [2]. Countries which have eradicated polio use killed injectable polio vaccine (IPV), mainly because oral polio vaccine (OPV) can result in paralytic poliomyelitis. In literature review, we could not find any reported cases of paralytic poliomyelitis following vaccination in Sri Lanka. We report a case of acute flaccid paralysis following immunisation with first dose of OPV.

\section{Case report}

A 3-month old girl presented with diminished movements of the body for 2 days duration. She also had fever, cough and diarrhoea. She did not have difficulties with feeding or breathing. She had her first dose of OPV, 32 days ago. She was previously well, without any features of immunodeficiency or neuromuscular disorders. Her birth was uncomplicated and she had normal development up to that time. She was the 3rd child of non-consanguineous parents.

Physical examination revealed an alert baby with normal facial expressions. She was markedly floppy. Asymmetrical weakness was observed in the upper limbs with grade 3 and 1 muscle power respectively in right and left sides. Lower limbs were completely paralysed. Deep tendon reflexes were absent in all limbs. Apart from a hoarse cry she did not have features of cranial nerve involvement. She responded to pain and other somatic stimuli. She had normal respiration, blood preassure and sphincter functions.

Her serum electrolytes, calcium, urea, and glucose were normal. Complete blood count revealed; haemoglobin - $10.9 \mathrm{~g} / \mathrm{dl}$, white cell count $10,400 / \mu \mathrm{l}(20 \%$ neutrophils and $68 \%$ lymphocytes) and platelets $571,000 / \mu 1$. Blood picture showed evidence of a viral infection. C-reactive protein was negative and the blood culture was sterile. Serum creatine kinase was $35.1 \mathrm{u} / 1$ (normal 24-190u/l). Nerve conduction study done on 6th day revealed normal peripheral nerve conduction velocity but absent $F$ waves, suggestive of a diffuse anterior horn cell disease. Repeat test in 1 week showed same results. Cerebrospinal fluid analysis on 12th day showed high protein content (146 $\mathrm{mg} / \mathrm{dl}$ ) without cellular pleocytosis. MRI scan of the spine was normal. Vaccine strain poliovirus was isolated from two stool specimens, obtained 6-7 days after the onset of paralysis.

Clinical diagnosis of acute flaccid paralysis (AFP), possibly due to OPV was made and baby was managed symptomatically. Physiotherapy was initiated in the second week. Review after 3 weeks revealed persistent weakness in all 4 limbs with a slight improvement of muscle power in the right upper limb. Four months after the disease onset, lower limb flaccid paralysis was persisting with only a flicker of movements in toes. Muscle power in upper limbs had improved to grade 4 .

\section{Discussion}

Although there is no standard case definition for vaccine-associated paralytic poliomyelitis (VAPP), it is generally defined as a case with AFP in whom vaccinestrain of poliovirus is isolated from stool samples and there is no other explanation for the AFP. The onset of paralysis should be within 4-40 days after receiving OPV and the residual weakness should persist for more than 60 days after its onset $[3,4]$. This patient fulfils these criteria, with AFP starting within 40 days of vaccination and persisting over 60 days, asymmetrical paralysis without sensory involvement, neurophysiologic features suggestive of anterior horn cell disease and isolation of vaccine type poliovirus from the stools. There were no other explanations for this infant's presentation. Therefore this is the first reported case of possible VAPP from Sri Lanka in the medical literature.

When a significant adverse reaction following vaccination is reported, it will have a major impact on immunisation programme of the country. There was a marked reduction in vaccine uptake in UK, following the paper published on possible association between autism and MMR vaccine [5]. In our country we had similar

${ }^{1}$ Department of Paediatrics, Faculty of Medicine, University of Kelaniya, Sri Lanka.

Correspondence: PP, e-mail: <priyanthaprr@gmail.com>. Received 30 June and revised version accepted 13 October 2011. Competing interests: none declared. 
experiences following unfortunate events that occured related to pentavalent and rubella vaccines. This is why we report this case as a possible case of VAPP, though it fulfills all criteria for a VAPP.

OPV induces gut associated immunity ( $\operatorname{IgA})$ as well as IgM and IgG in blood. However, OPV is known to cause paralytic poliomyelitis in 0.3 cases per million doses, by 3 different mechanisms [2]. Firstly in vaccine recipients it can cause VAPP, secondly it may result in transmissible and neurovirulent circulating vaccine-derived poliovirus (cVDPV) strains and finally immunodeficient vaccinees can get VAAP. These individuals excrete 'immunodeficient VDPV' (iVDPV) for several decades posing a threat to non-immune contacts [6].

The pathophysiology of poliomyelitis due to vaccine strain is similar to that of wild virus. Direct cytopathic effect of virus causes neuronal death, resulting in flaccid paralysis of muscles innervated by them. Neuronal involvement is patchy and asymmetrical, resulting in asymmetrical paralysis. Some affected neurons recover, explaining the improvement seen in muscle function during the early stages.

Due to this risk, countries which have erradicated polio have replaced OPV with IPV in their immunisation programmes [7]. No case of VAPP was reported In USA after substituting OPV by IPV [8]. However, for Sri Lanka, there are two major concerns regarding replacing OPV with IPV. First is the cost, but a cost analysis done has shown that cost would be same in the future [9]. The other concern is prevalence of polio in neighbouring countries. WHO recommends OPV in countries which have not eradicated polio. This is because herd immunity produced by OPV is important in eradication of polio.

However, in Sri Lanka an IPV is licenced and used in the private sector, while OPV is used in the National Immunisation Programme (NIP). One can argue if IPV is allowed to be used in private sector, why not include it in
NIP. The main objective of reporting this case is to make this information available for any future decision making regarding this issue. Authors have no conflicts of interest in this regard.

\section{References}

1. World Health Organization. Polio Eradication: Final Strategy. World Health Organization - Regional Office for south-east Asia 2003. Colombo, Sri Lanka.

2. Palihawadana P. Poliomyelitis vaccine. In: Wimalarathna O, Weerasinghe A, Jayasuriya L, eds. SLMA Guidelines and Information on Vaccines. Colombo: Sri Lanka Medical Association. 2008: 65-8.

3. Pan American Health Organization. Norms and standards in epidemiology: guidelines for epidemiological surveillance. Epidemiological Bulletin 1999; 20: 12-3.

4. Sun Jun Kim, Sung Han Kim, Young Mee Jee, Jung Soo Kim. Vaccine-associated paralytic poliomyelitis: a case report of flaccid monoparesis after oral polio vaccine. Journal of Korean Medical Science 2007; 22: 362-4.

5. JW Lee, B Melgaard, CJ Clements, et al. (Correspondence)Autism, inflammatory bowel disease and MMR vaccine. Lancet 1998; 351: 905.

6. Minor P. Vaccine-derived poliovirus (VDPV): impact on poliomyelitis eradication. Vaccine 2009; 27: 2649-52.

7. World Health Organization. Introduction of inactivated poliovirus vaccine into oral poliovirus vaccine-using countries. Weekly Epidemiological Record 2003; 78: 241-50.

8. Alexander L, Seward J, Santibanez T, et al. Vaccine policy changes and epidemiology of poliomyelitis in the United States. Journal of American Medical Association 2004; 292: 1696-1701.

9. Sangrujee N, Cáceres V, Cochi S. Cost analysis of post-polio certification immunization policies. Bulletin of the World Health Organization 2004; 82: 9-15. 